\title{
INTRABEAM SCATTERING ANALYSIS OF ATF BEAM MEASUREMENTS*
}

\author{
K.L.F. Bane, SLAC, Stanford, CA 94309, USA \\ H. Hayano, K. Kubo, T. Naito, T. Okugi, J. Urakawa, KEK, Tsukuba, Japan \\ 1 INTRODUCTION

\section{IBS CALCULATIONS}

In future e+e- linear colliders, such as the JLC/NLC, damping rings are needed to generate beams of intense bunches with very low emittances. The Accelerator Test Facility (ATF)[1] at KEK is a prototype for such damping rings. In April 2000 the single bunch energy spread, bunch length, and horizontal and vertical emittances of the beam in the ATF were all measured as functions of current, and all exhibited significant growth[2][3]. We want to know if the results agree with intrabeam scattering (IBS) theory.

In the ATF as it is now, running below design energy and with the wigglers turned off, IBS is relatively strong for an electron machine. It is an effect that couples all dimensions of the beam. Unique at the ATF is that the beam energy spread, an especially important parameter in IBS theory, can be measured to an accuracy of a few percent. The bunch length measurement is important since at the ATF potential well bunch lengthening is significant[3]. Evidence that we are truly seeing IBS at the ATF include (see also Ref. [4]): (1) when moving onto the coupling resonance, the normally large energy spread growth with current becomes negligibly small; (2) if we decrease the vertical emittance using dispersion correction, the energy spread increases.

Calculations of IBS tend to use the equations of Piwinski[5] (P) or of Bjorken and Mtingwa[6] (B-M). Both approaches solve the local, two-particle Coulomb scattering problem under certain assumptions, but the results appear to be different. The B-M result is thought to be the more accurate of the two[7]. Another, simpler formulation is due to Raubenheimer (R)[8]. Also found in the literature is a more complicated result that allows for $x-y$ coupling[9], and a recent formulation that includes effects of the impedance[10]. An optics computer program that solves IBS, using the B-M equations, is SAD[11].

Calculations of IBS tend to be applied to proton or heavy ion storage rings, where effects of IBS are normally more pronounced. Examples of comparisons of IBS theory with measurement can be found for proton[12],[13] and electron machines[14],[15]. In such reports, although good agreement is often found, the comparison/agreement is usually not complete (e.g. in Ref. [12] growth rates agree reasonably well in the longitudinal and horizontal, but completely disagree in the vertical) and/or a fitting or "fudge" factor is needed to get agreement (e.g. Ref. [15]).

In the present report we briefly describe the IBS formulations, apply them to ATF parameters, and finally compare calculations with the data of April 2000.

\footnotetext{
*Work supported by the Department of Energy, contract DE-AC03$76 \mathrm{SF} 00515$
}

We begin by sketching the general method of calculating the effect of IBS in a storage ring (see, e.g. Ref. [5]). Let us first assume that there is no $x-y$ coupling.

Let us consider the IBS growth rates in energy $p$, in the horizontal $x$, and in the vertical $y$ to be defined as

$$
\frac{1}{T_{p}}=\frac{1}{\sigma_{p}} \frac{d \sigma_{p}}{d t}, \quad \frac{1}{T_{x}}=\frac{1}{\epsilon_{x}^{1 / 2}} \frac{d \epsilon_{x}^{1 / 2}}{d t}, \quad \frac{1}{T_{y}}=\frac{1}{\epsilon_{y}^{1 / 2}} \frac{d \epsilon_{y}^{1 / 2}}{d t} .
$$

Here $\sigma_{p}$ is the rms (relative) energy spread, $\epsilon_{x}$ the horizontal emittance, and $\epsilon_{y}$ the vertical emittance. In general, the growth rates are given in both $\mathrm{P}$ and $\mathrm{B}-\mathrm{M}$ theories in the form (for details, see Refs. [5],[6] ${ }^{1}$ ):

$$
\frac{1}{T_{i}}=\left\langle f_{i}\right\rangle
$$

where subscript $i$ stands for $p, x$, or $y$. The functions $f_{i}$ are integrals that depend on beam parameters, such as energy and phase space density, and lattice properties, including dispersion ( $y$ dispersion, though not originally in B-M, can be added in the same manner as $x$ dispersion); the brackets \langle\rangle mean that the quantity is averaged over the ring.

From the $1 / T_{i}$ we obtain the steady-state properties for machines with radiation damping:

$$
\epsilon_{x}=\frac{\epsilon_{x 0}}{1-\tau_{x} / T_{x}}, \epsilon_{y}=\frac{\epsilon_{y 0}}{1-\tau_{y} / T_{y}}, \sigma_{p}^{2}=\frac{\sigma_{p 0}^{2}}{1-\tau_{p} / T_{p}},
$$

where subscript 0 represents the beam property due to synchrotron radiation alone, i.e. in the absence of IBS, and the $\tau_{i}$ are synchrotron radiation damping times. These are 3 coupled equations since all 3 IBS rise times depend on $\epsilon_{x}$, $\epsilon_{y}$, and $\sigma_{p}$. Note that a 4 th equation, the relation between bunch length $\sigma_{s}$ and $\sigma_{p}$, is also implied; generally this is taken to be the nominal (zero current) relation.

The best way to solve Eqs. 3 is to convert them into 3 coupled differential equations, such as is done in e.g. Ref. [15], and solve for the asymptotic values. For example, the equation for $\epsilon_{y}$ becomes

$$
\frac{d \epsilon_{y}}{d t}=-\frac{\left(\epsilon_{y}-\epsilon_{y 0}\right)}{\tau_{y}}+\frac{\epsilon_{y}}{T_{y}}
$$

and there are corresponding equations for $\epsilon_{x}$ and $\sigma_{p}^{2}$.

Note that:

- For weak coupling, we add the term $-\kappa \epsilon_{x}$, with $\kappa$ the coupling factor, into the parenthesis of the $\epsilon_{y}$ differential equation, Eq. 4.

${ }^{1}$ We believe that the right hand side of Eq. 4.17 in B-M (with $\sigma_{\eta}$ equal to our $\sqrt{2} \sigma_{p}$ ) should be divided by $\sqrt{2}$, in agreement with the recent derivation of Ref. [10]. 
- A conspicuous difference between the $\mathrm{P}$ and B-M results is their dependence on dispersion $\eta$ : for $\mathrm{P}$ the $f_{i}$ depend on it only through $\eta^{2}$; for B-M, through $\left[\eta^{\prime}+\beta^{\prime} \eta /(2 \beta)\right]$ and the dispersion invariant $\mathcal{H}=$ $\bar{\gamma} \eta^{2}+2 \alpha \eta \eta^{\prime}+\beta \eta^{\prime 2}$, with $\alpha, \beta, \bar{\gamma}$ Twiss parameters.

- We approximate the effect of potential well bunch lengthening in our IBS calculations by adding a multiplicative factor $f_{p w}(I)$ [ $I$ is current], obtained from measurements, to the equation relating $\sigma_{s}$ to $\sigma_{p}$.

- The results include a so-called Coulomb log factor, $\ln \left(b_{\max } / b_{\min }\right)$, with $b_{\max }, b_{\min }$ maximum, minimum impact parameters, quantities which are not well defined. We take $b_{\max }=\sigma_{y} ; b_{\min }=r_{0} c^{2} /\left\langle v_{x}^{2}\right\rangle$ $=r_{0} \beta_{x} /\left(\gamma^{2} \epsilon_{x}\right)$, with $r_{0}$ the classical electron radius, $v_{x}$ the transverse velocity in the rest frame, and $\gamma$ the energy factor. For the ATF, $\ln ()=16.0$.

- The IBS bunch distributions are not Gaussian, and tail particles can be overemphasized in these solutions. We are interested in core sizes, which we estimate by eliminating interactions with collision rates less than the synchrotron radiation damping rate[17]. We can approximate this in the Coulomb $\log$ term by letting $\pi b_{\min }^{2}\left\langle\left|v_{x}\right|\right\rangle\langle n\rangle=1 / \tau$, with $n$ the particle density in the rest frame[16]; or $b_{\min }=$ $\sqrt{4 \pi \sigma_{x} \sigma_{y} \sigma_{z} /[N c \tau]}\left(\beta_{x} / \epsilon_{x}\right)^{1 / 4}$, with $N$ the bunch population. For the ATF with this cut, $\ln ()=13.9$.

\subsection{Emittance Growth}

An approximation to Eqs. 2, valid for typical, flat electron beams is due to Raubenheimer (R) [8],[18]: ${ }^{2}$

$$
\begin{aligned}
\frac{1}{T_{p}} & \approx \frac{r_{0}^{2} c N}{32 \gamma^{3} \epsilon_{x} \epsilon_{y} \sigma_{s} \sigma_{p}^{2}}\left(\frac{\epsilon_{x} \epsilon_{y}}{\left\langle\beta_{x}\right\rangle\left\langle\beta_{y}\right\rangle}\right)^{1 / 4} \ln \left(\frac{\left\langle\sigma_{y}\right\rangle \gamma^{2} \epsilon_{x}}{r_{0}\left\langle\beta_{x}\right\rangle}\right) \\
\frac{1}{T_{x, y}} & \approx \frac{\sigma_{p}^{2}\left\langle\mathcal{H}_{x, y}\right\rangle}{\epsilon_{x, y}} \frac{1}{T_{p}} .
\end{aligned}
$$

If the vertical emittance is due only to vertical dispersion then[8]

$$
\epsilon_{y 0} \approx \mathcal{J}_{\epsilon}\left\langle\mathcal{H}_{y}\right\rangle \sigma_{p 0}^{2}
$$

with $\mathcal{J}_{\epsilon}$ the energy damping partition number. We can solve Eqs. 3,5,6 to obtain the steady-state beam sizes. Note that once the vertical orbit-and therefore $\left\langle\mathcal{H}_{y}\right\rangle$-is set, $\epsilon_{y 0}$ is also determined.

Following an argument in Ref. [8] we can obtain a relation between the expected vertical and horizontal emittance growth due to IBS in the presence of random vertical dispersion: The beam momentum in the longitudinal plane is much less than in the transverse planes. Therefore, IBS will first heat the longitudinal plane; this, in turn, increases the transverse emittances through dispersion (through $\mathcal{H}$ ), like synchrotron radiation (SR) does. One difference between IBS and SR is that IBS increases the emittance everywhere, and SR only in bends. We can write

$$
\frac{\epsilon_{y 0}}{\epsilon_{x 0}} \approx \frac{\mathcal{J}_{x}\left\langle\mathcal{H}_{y}\right\rangle_{b}}{\mathcal{J}_{y}\left\langle\mathcal{H}_{x}\right\rangle_{b}} \quad, \quad \frac{\epsilon_{y}-\epsilon_{y 0}}{\epsilon_{x}-\epsilon_{x 0}} \approx \frac{\mathcal{J}_{x}\left\langle\mathcal{H}_{y}\right\rangle}{\mathcal{J}_{y}\left\langle\mathcal{H}_{x}\right\rangle},
$$

${ }^{2}$ Our equation for $1 / T_{p}$ is twice as large as Eq. 2.3.5 of Ref. [8]. where $\mathcal{J}_{x, y}$ are damping partition numbers, and \langle\rangle$_{b}$ means averaging is only done over the bends. For vertical dispersion due to errors we expect $\left\langle\mathcal{H}_{y}\right\rangle_{b} \approx\left\langle\mathcal{H}_{y}\right\rangle$. Therefore,

$$
r_{\epsilon} \equiv \frac{\left(\epsilon_{y}-\epsilon_{y 0}\right) / \epsilon_{y 0}}{\left(\epsilon_{x}-\epsilon_{x 0}\right) / \epsilon_{x 0}} \approx \frac{\left\langle\mathcal{H}_{x}\right\rangle_{b}}{\left\langle\mathcal{H}_{x}\right\rangle},
$$

which, for the ATF is 1.6. If, however, there is only $x-y$ coupling, $r_{\epsilon}=1$; if there is both vertical dispersion and coupling, $r_{\epsilon}$ will be between $\left\langle\mathcal{H}_{x}\right\rangle_{b} /\left\langle\mathcal{H}_{x}\right\rangle$ and 1 .

\subsection{Numerical Comparison}

Let us compare the results of the P, B-M, and R methods when applied to the ATF beam parameters and lattice, with vertical dispersion and no $x-y$ coupling. We take: current $I=3.1 \mathrm{~mA}$, energy $E=1.28 \mathrm{GeV}$, $\sigma_{p 0}=5.44 \times 10^{-4}, \sigma_{s 0}=5.06 \mathrm{~mm}$ (for an rf voltage of $300 \mathrm{kV}$ ), $\epsilon_{x 0}=1.05 \mathrm{~nm}, \tau_{p}=20.9 \mathrm{~ms}, \tau_{x}=18.2 \mathrm{~ms}$, and $\tau_{y}=29.2 \mathrm{~ms} ; f_{p w}=1$. The ATF circumference is $138 \mathrm{~m}, \mathcal{J}_{\epsilon}=1.4,\left\langle\beta_{x}\right\rangle=3.9 \mathrm{~m},\left\langle\beta_{y}\right\rangle=4.5 \mathrm{~m}$, $\left\langle\eta_{x}\right\rangle=5.2 \mathrm{~cm}$ and $\left\langle\mathcal{H}_{x}\right\rangle=2.9 \mathrm{~mm}$. To generate vertical dispersion we randomly offset magnets by $15 \mu \mathrm{m}$, and then calculate the closed orbit using SAD. For our seed we find that the rms dispersion $\left(\eta_{y}\right)_{r m s}=7.4 \mathrm{~mm}$, $\left\langle\mathcal{H}_{y}\right\rangle=17 \mu \mathrm{m}$, and $\epsilon_{y 0}=6.9 \mathrm{pm}$ (in agreement with Eq. 6). For consistency between the methods we here take $\ln ()=\ln \left[\left\langle\sigma_{y}\right\rangle \gamma^{2} \epsilon_{x} /\left(r_{0}\left\langle\beta_{x}\right\rangle\right)\right]=16$.

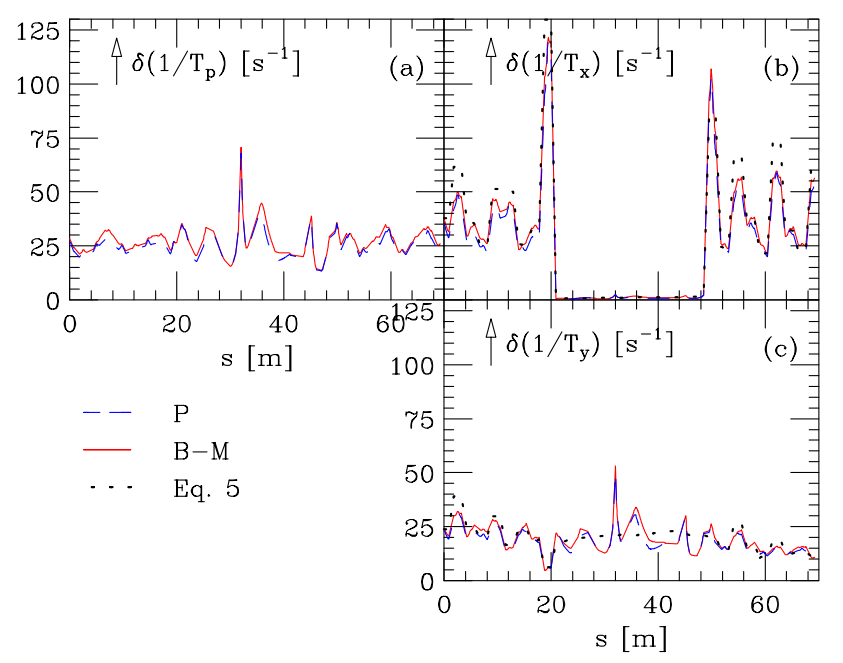

Figure 1: Differential growth rates over $1 / 2$ the ATF, as obtained by modified Piwinski and Bjorken-Mtingwa.

Performing the calculations, we find that the growth rates in $p$ and $x$ agree well between the two methods; the vertical rate, however, does not, with the $\mathrm{P}$ result $25 \%$ low. From the arguments of Sec. 2.1, we might expect that we can improve the $\mathrm{P}$ calculation if we replace $\eta_{x, y}^{2} / \beta_{x, y}$ by $\mathcal{H}_{x, y}$. Doing this we find that, indeed, the three growth rates now agree reasonably well with the B-M results. Fig. 1 displays the differential IBS growth rates over half the ring (the periodicity is 2 ), as obtained by the modified $\mathrm{P}$ and $\mathrm{B}-\mathrm{M}$ methods. As for the $1 / T_{i}$, the average values of these functions, the $\mathrm{P}$ results are all slightly 
low, by $6 \%$. The B-M method gives: $1 / T_{p}=27.0 \mathrm{~s}^{-1}$, $1 / T_{x}=26.0 \mathrm{~s}^{-1}, 1 / T_{y}=19.4 \mathrm{~s}^{-1} ; \sigma_{p} / \sigma_{p 0}=1.52$, $\epsilon_{x} / \epsilon_{x 0}=1.90, \epsilon_{y} / \epsilon_{y 0}=2.30$. The emittance ratio of Eq. 8 is $r_{\epsilon}=1.44$, close to the expected 1.6.

The dots in Fig. 1b,c give the differential rates corresponding to Eq. 5, and we see that the agreement also is good. The growth rates in $(p, x, y)$ are $(27.0,26.4,19.3) \mathrm{s}^{-1}$, the relative growths in $\left(\sigma_{p}, \epsilon_{x}, \epsilon_{y}\right)$ are $(1.51,1.92,2.29)$.

\section{COMPARISON WITH MEASUREMENT}

The parameters $\sigma_{p}, \sigma_{s}, \epsilon_{x}$, and $\epsilon_{y}$ were measured in the ATF as functions of current over a short period of time at rf voltage $V_{c}=300 \mathrm{kV}$. Energy spread was measured on a screen at a dispersive region in the extraction line (Fig. 2a); bunch length with a streak camera in the ring (Fig. 2b). The curves in the plots are fits that give the expected zero current result. Emittances were measured on wire monitors in the extraction line (the symbols in Fig. 3b-c; note that the symbols in Fig. 3a reproduce the fits to the data of Fig. 2). We expect the random component of errors in $y$ to be 5$10 \%$, and less in $x$. However, since $\epsilon_{y}$ is small, one can imagine that its measurement can easily be corrupted by factors such as roll or dispersion in the extraction line. We see that $\epsilon_{x}$ appears to grow by $\sim 85 \%$ by $I=3 \mathrm{~mA} ; \epsilon_{y}$ begins at about 1.0-1.2\% of $\epsilon_{x 0}$, and then grows to about $.03 \epsilon_{x 0}$; i.e. $r_{\epsilon}=1.8-2.4$. If we are vertical dispersion dominated, with $\left(\eta_{y}\right)_{r m s}=10 \mathrm{~mm}$ and $\epsilon_{y 0} \approx .012 \epsilon_{x 0}$, then the data nearly satisfies Eq. $8, r_{\epsilon} \approx 1.6$. (However, normally, the residual dispersion at the ATF is kept to $\left(\eta_{y}\right)_{r m s}=3-5 \mathrm{~mm}$.) If we are coupling dominated we see that $r_{\epsilon} \approx 1$ is not well satisfied by the data.
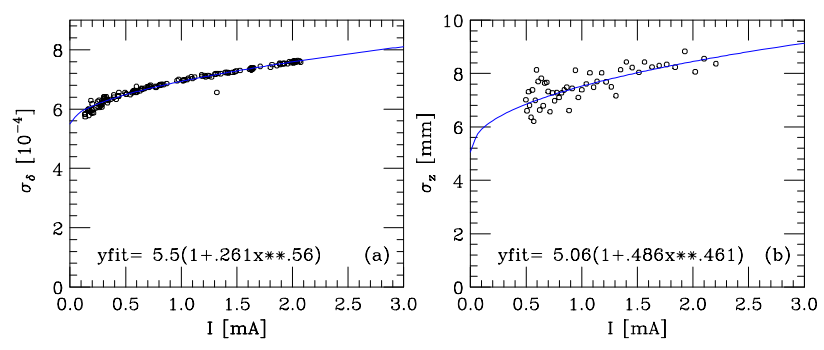

Figure 2: Measurements of energy spread (a) and bunch length (b), with $V_{c}=300 \mathrm{kV}$.

Let us compare B-M calculations with the data. Here we take $f_{p w}$ as given by the measurements, and take $\ln ()=$ 14. At $I=3 \mathrm{~mA}$ we adjust $\epsilon_{y 0}$ until the calculated $\sigma_{p}$ agrees with the measurement. In Fig. 3 we give examples: (1) with vertical dispersion only, with $\left(\eta_{y}\right)_{r m s}=7.0 \mathrm{~mm}$ and $\epsilon_{y 0}=6.3 \mathrm{pm}$ (solid); (2) coupling dominated with $\left(\eta_{y}\right)_{\text {rms }}=3 \mathrm{~mm}$ and $\epsilon_{y 0}=8.7 \mathrm{pm}$ (dashes); (3) increasing the strength of IBS by increasing $\ln ()$ by $35 \%$ : i.e. letting $\ln ()=19$, for the coupling dominated example with $\left(\eta_{y}\right)_{r m s}=3 \mathrm{~mm}$ and $\epsilon_{y 0}=14.7 \mathrm{pm}$ (dotdash); (4) same as Ex. 2 but assuming a small amount of $\epsilon_{y}$ measurement error, i.e. adding $0.6 \% x-y$ coupling error (the dots).

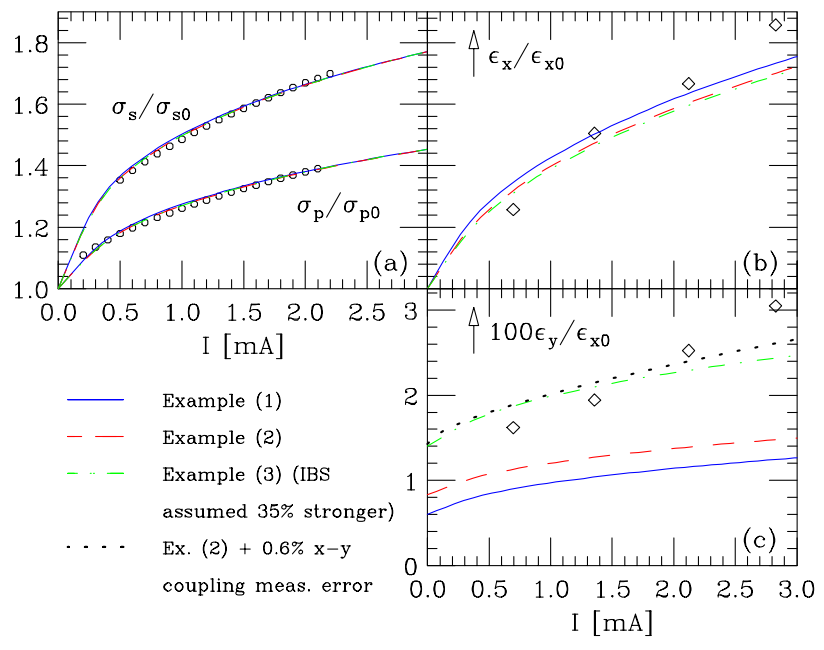

Figure 3: ATF measurement data (symbols) and IBS theory fits (the curves). The symbols in (a) give the smooth curve fits to the measured data of Fig. 2.

In conclusion, we have derived a simple relation for growth rates of emittances due to IBS. We have found that IBS calculations following Piwinski (with $\eta^{2} / \beta$ replaced by $\mathcal{H}$ ), Bjorken-Mtingwa, and a formula due to Raubenheimer, when applied to the ATF, all agree well. Comparing the Bjorken-Mtingwa calculations with ATF measurements of April 2000, we found reasonably good agreement in energy spread and horizontal emittance dependence on current. The vertical emittance measurement, however, implies that either: there is error in the measurement (equivalent to the introduction of a $0.6 \% x-y$ coupling error), or intrabeam scattering is stronger than predicted (35\% stronger in growth rates). In addition, the slope of the vertical emittance dependence on current is steeper than predicted.

We thank A. Piwinski, K. Oide, and C. Steier for helpful discussions on IBS.

\section{REFERENCES}

[1] F. Hinode, editor, KEK Internal Report 95-4 (1995).

[2] J. Urakawa, Proc. EPAC2000, Vienna (2000) p. 63.

[3] K. Bane, et al, presented at ISEM2001, Tokyo, May 2001.

[4] K. Kubo, "Recent Progress in the Accelerator Test Facility at KEK," presented at HEACC2001, Tsukuba, March 2001.

[5] A. Piwinski, in A. Chao and M. Tigner, eds., Handbook of Accelerator Physics and Engineering (1999), p. 125.

[6] J. Bjorken, S. Mtingwa, Particle Accel. 13 (1983) 115.

[7] A. Piwinski, private communication.

[8] T. Raubenheimer, PhD Thesis, SLAC-387, Sec. 2.3.1, 1991.

[9] A. Piwinski, CERN Accelerator School (1991) p. 226.

[10] Marco Venturini, "Intrabeam Scattering and Wake Field Forces in Low Emittance Electron Rings," this conference.

[11] K. Oide, SAD User's Guide.

[12] M. Conte, M. Martini, Particle Accelerators 17 (1985) 1.

[13] L.R. Evans and J. Gareyte, PAC85, IEEE Trans. in Nuclear Sci. NS-32 No. 5 (1985) 2234.

[14] C.H. Kim, Proc. PAC97, Vancouver (1997) p. 790.

[15] C.H. Kim, LBL-42305, September 1998.

[16] K. Oide, Proc. of SAD Workshop, KEK (1998) p. 125.

[17] T. Raubenheimer, Particle Accelerators 45 (1994) 111.

[18] A. Piwinski, Proc. SSC Workshop, Ann Arbor (1983) p. 59. 